\title{
Surface rheology of monolayers of phospholipids and cholesterol measured with axisymmetric drop shape analysis
}

\author{
Marcel Vrânceanu ${ }^{\mathrm{a}, \mathrm{b}}$, Karin Winkler ${ }^{\mathrm{a}}$, Hermann Nirschl ${ }^{\mathrm{b}}$, Gero Leneweit ${ }^{\mathrm{a}, *}$ \\ ${ }^{a}$ Carl Gustav Carus-Institute, Am Eichhof 30, 75223 Niefern-Öschelbronn, Germany \\ ${ }^{\mathrm{b}}$ University of Karlsruhe (TH), Institute of Mechanical Process Engineering and Mechanics, Am Forum 8, 76128 Karlsruhe, Germany
}

Received 25 December 2006; received in revised form 4 June 2007; accepted 8 June 2007

Available online 14 June 2007

\begin{abstract}
Surface rheology of monolayers of a saturated phospholipid (dipalmitoylphosphatidylcholine, DPPC), an unsaturated phospholipid (dioleoylphosphatidylcholine, DOPC) and cholesterol is studied with axisymmetric drop shape analysis at the argon/water interface. Measurement techniques for lipids are described in detail. Profile analysis tensiometry (PAT) is used to determine the film pressure $\Pi$, surface elasticity and surface dilational viscosity of monolayers upon sinusoidal oscillations of the drop surface for various amplitudes $a$ and frequencies $f$ to assess their dependence on these dynamic parameters. It is shown that surface dilational viscosity strongly depends on the frequency and decreases by a factor 2-5 with increasing $f$ in the considered range. Dilational viscosity is higher the more the monolayer approaches a relaxed state. Thus, the molecular interactions are stronger in the relaxed than in the stressed state. Surface elasticity is much less dependent on dynamic conditions. For DPPC a minimum of the dynamic surface elasticity is found for $f=12.5 \mathrm{mHz}$ (at $\Pi=17.5 \mathrm{mN} \mathrm{m}^{-1}$ ) which coincides well with the relaxation frequencies measured in stress relaxation experiments. The dynamic surface elasticity of DPPC exhibits a plateau in the range $13.5 \mathrm{mN} \mathrm{m}^{-1} \leq \Pi \leq 27 \mathrm{mN} \mathrm{m}^{-1}$ in good coincidence with the phase boundaries of the coexistence region of micron-sized liquid crystalline domains surrounded by a fluid monolayer phase. In equilibrium measurements ( $\Pi / A$-isotherms) a plateau of the film pressure is seen at the lower bound and a break at the upper bound of the coexistence region. Film pressure/area isotherms produced by PAT and a Langmuir film balance closely coincide as is shown in a comparison to literature values. However, the surface elasticities measured dynamically with oscillating surfaces widely deviate from those derived from isotherms in the case of DPPC and cholesterol, whereas for DOPC very good agreement can be found.
\end{abstract}

(C) 2007 Elsevier B.V. All rights reserved.

Keywords: DPPC; DOPC; Profile analysis tensiometer; Surface dilational viscosity; Surface elasticity

\section{Introduction}

Rheological properties of phospholipid monolayers are of fundamental importance for the physiology of breathing, since the fluid layer on the alveolar lining is covered by monolayers consisting of almost $90 \%$ phospholipids [1]. Consequences of surface rheology on respiratory performance have been discussed in detail [2-5]. Further, the surface rheology of phos-

Abbreviations: DPPC, dipalmitoylphosphatidylcholine; DPPG, dipalmitoylphosphatidylglycerol; DOPC, dioleoylphosphatidylcholine; ADSA, axisymmetric drop shape analysis; PAT, profile analysis tensiometer; THD, total harmonic distortion

* Corresponding author. Tel.: +49 7233 68443; fax: +49 723368413 .

E-mail addresses: marcel.vranceanu@carus-institut.de (M. Vrânceanu), winkler@abnoba.de (K. Winkler), hermann.nirschl@mvm.uni-karlsruhe.de (H. Nirschl), gero.leneweit@ carus-institut.de (G. Leneweit). pholipids effects the properties of many emulsion systems, influencing emulsion stability [6], droplet deformation and suspension rheology [7]. As the physicochemical properties of monolayers are similar to those of bilayers, monolayers and bilayers of phospholipids mixed with cholesterol have been studied extensively both in the context of cell physiology [8,9] and artificial bilayer systems like liposomes [10].

There are fundamental differences in the rheology and phase formation of phospholipids consisting of either saturated or unsaturated fatty acids. To characterize them, two ubiquitous phospholipids with a phosphatidylcholine headgroup, but either saturated (dipalmitoylphosphatidylcholine, DPPC) or monounsaturated fatty acids (dioleoylphosphatidylcholine, DOPC) were chosen for this study.

Various methods have been used to characterize rheology, phase separations and transitions of pure phospholipids, especially DPPC and DOPC, and their mixtures with cholesterol. 
Classically, film pressure versus area isotherms are produced on a Langmuir film balance, that allow to derive the dilational elasticity in thermodynamic equilibrium. Isotherms are reported for DPPC [4,11-23], DOPC [11,24-26], binary DPPC/cholesterol [15,27], binary DOPC/cholesterol [25,26], and ternary DOPC/DPPC/cholesterol mixtures [28]. To obtain the surface dilational viscosity and the dynamic surface elasticity, monolayers on a Langmuir film balance are studied with an oscillating barrier at various frequencies, which is described for DPPC [16,19,20] and DOPC [24]. Surface shear viscosity of DPPC was studied with a shear rheometer [19,20,29].

Area oscillations of drop or bubble surfaces can be performed with a profile analysis tensiometer (PAT) using the principle of axisymmetric drop shape analysis (ADSA). Here, monolayer compression and expansion is more homogeneously distributed over the whole area than on a Langmuir trough. ADSA was used to study DPPC [4,21,30] and other phospholipid monolayers at the air-water interface [3,4,21,30,31], chloroform/water interface [21,22,30], $n$-dodecane/water interface $[23,30]$ or dichloromethane/water interface [32]. Alternatively to a pendent drop, PAT can also be used to study a captive bubble under prescribed oscillations. This was performed for DPPC [2,11] and DOPC [11].

However, there is not very much information so far regarding the dependence of surface dilational viscosity and elasticity on dynamic parameters, i.e. frequency and amplitude of harmonic area oscillations. Wüstneck et al. [3] used PAT to study the stress relaxation process of a DPPG monolayer and the influence of oscillation frequency on the surface dilational viscosity and elasticity. The influence of oscillation amplitude was not studied systematically so far. Wüstneck et al. [3] observe that the rheological parameters determined from harmonic oscillations may depend on the amplitude. Therefore, we present a systematic study of the influence of the oscillation amplitude and frequency on dilational elasticity and viscosity and the distortions caused by higher harmonics. We quantitatively compare our results produced with PAT regarding the surface elasticity of DPPC, DOPC and cholesterol with those of other authors, who either used dynamic or static methods. With that aim, we compare film pressure/area isotherms of DPPC measured with PAT and with a Langmuir film balance to assess whether discrepancies stem from differences of the surface geometry or from the difference of dynamic or static conditions of measurement.

The surface rheological properties, phase transitions and separations of monolayers of DPPC-cholesterol and DOPC-cholesterol binary mixtures as functions of the film pressure will be presented in a further publication [33].

\subsection{Background}

ADSA allows to determine the surface tension $\sigma$, the film pressure $\Pi$, the surface dilational viscosity $\eta$ and the surface elasticity $\varepsilon$ of a monolayer present at the liquid-liquid or liquid-gas interface. The main principle is to determine the surface tension of a liquid from the shape of a pendent drop. The surface tension $\sigma$ is calculated by fitting the drop shape to the
Young-Laplace equation. The film pressure $\Pi=\sigma_{0}-\sigma_{\mathrm{L}}$ is the difference of the surface tension $\sigma_{0}$ of the pure subphase (water) and that with the lipid monolayer $\sigma_{\mathrm{L}}$.

The response of the surface tension $\sigma$ to harmonic area oscillations gives information about the rheological properties of monolayers. The surface elasticity $\varepsilon$ in thermodynamic equilibrium is given by

$\varepsilon=\frac{\mathrm{d} \sigma}{\mathrm{d} \ln A}=\frac{\mathrm{d} \sigma}{\mathrm{d} A} A$

where $\mathrm{d} \sigma$ describes the infinitesimal surface tension gradients upon a relative variation of the area $A$. It was shown by Loglio et al. [34] that a complex elasticity modulus $E$ can be determined by

$E(\mathrm{i} \omega)=\frac{\mathcal{F}\{\delta \sigma(t)\}}{\mathcal{F}\{\delta \ln A(t)\}}$

where $\mathcal{F}$ denotes the Fourier transformation operator, $\omega=2 \pi f$ the circular frequency, $t$ the time, $\delta \ln A(t)$ the variation of the relative surface area with the frequency $f$ and $\delta \sigma(t)$ is the surface tension response. Eq. (2) is only applicable when the time dependent variations are small enough to produce a linear relation between $\delta \ln A(t)$ and $\delta \sigma(t)$. The complex elasticity modulus can be displayed as

$$
\begin{aligned}
& E(\mathrm{i} \omega)=E^{\prime}(\omega)+\mathrm{i} E^{\prime \prime}(\omega)=|E| \exp (\mathrm{i} \theta) \text { with } \\
& |E|=\sqrt{E^{\prime 2}+E^{\prime \prime 2}}, \quad \tan \theta=\frac{E^{\prime \prime}}{E^{\prime}}
\end{aligned}
$$

where $\theta$ is the phase angle between the harmonic area oscillation and surface tension response. From Eq. (3) the dynamic surface elasticity $\varepsilon$ and the dilational viscosity $\eta$ can be derived:

$\varepsilon(\omega)=E^{\prime}(\omega) ; \quad \eta(\omega)=\frac{E^{\prime \prime}(\omega)}{\omega}=\frac{\varepsilon \tan \theta}{\omega}$

\section{Materials and methods}

\subsection{Materials}

1,2-Dioleoyl-sn-glycero-3-phosphocholine (DOPC) and 1,2-dipalmitoleoyl-sn-glycero-3-phosphocholine (DPPC) were obtained from Lipoid (Ludwigshafen, Germany), and cholesterol (standard for chromatography) from Sigma (Taufkirchen, Germany). As spreading solvent chloroform from Roth (Karlsruhe, Germany) was used. All materials had an estimated purity of more than $99 \%$ and were used without further purification. Bidistilled water with the quality for injectable drugs was used as subphase. All experiments were performed at ambient temperature, in the range $24.2 \pm 3{ }^{\circ} \mathrm{C}$, the precise temperature is given in the legend of each figure. To avoid lipid oxidation, argon was flowing constantly through the measurement chamber. The argon flow was saturated with water vapour by bubbling through water in a bottle. Water evaporation of the drop subphase was compensated by the dosing system, see Section 2.2. 


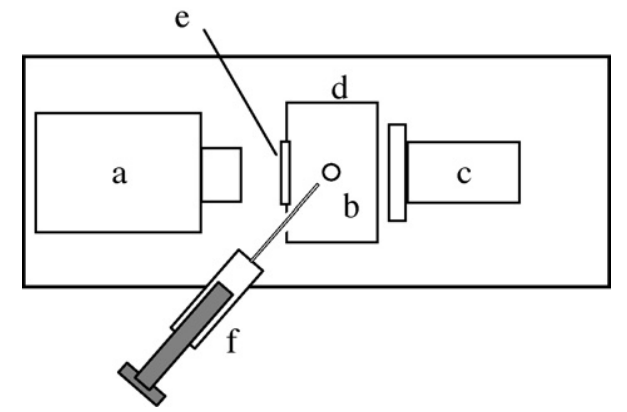

Fig. 1. Experimental setup: (a) CCD camera; (b) pendent drop; (c) light source; (d) measurement chamber; (e) optical glass; (f) $0.5 \mu 1$ syringe.

\subsection{Surface rheology measurements}

The dynamic surface properties of monolayers were measured with a pendent drop tensiometer PAT1 from Sinterface (Berlin, Germany). The method of measurement is described in [31]. A schematic drawing of the tensiometer seen from above is presented in Fig. 1. A CCD camera (a) records the shape of the pendent drop (b) which is illuminated with a light source (c). The home-made measurement chamber (d) has an optical glass (e) on the CCD camera side, and a small orifice for the needle of the microliter syringe (f). The syringe is placed on a holder, which can be moved in all directions and has a micrometer-adjustable endpoint.

The pendent drop was formed at the tip of a stainless steel capillary with an exterior diameter of $0.7 \mathrm{~mm}$. A monolayer was deposited with the microliter syringe at the drop surface as described in Section 2.4. After deposition, the monolayer film pressure $\Pi$ was followed in time at a constant drop surface $A$. Once equilibrium was approached (i.e. $\Delta \Pi \mathrm{mN} \mathrm{m}^{-1} \leq 0.1 \mathrm{mN} \mathrm{m}^{-1}$ within $5 \mathrm{~min}$ ), measurement of $\varepsilon$ and $\eta$ was started, i.e. $\Pi$ of an oscillating drop was recorded. Data were analysed with a Fourier transformation function for a generally 5 periods. The drop oscillation was performed with the tensiometer automatic dosing system, which varied $A(t)$ following a sinusoidal function of a prescribed amplitude and frequency:

$A(t)=A_{0}+a_{1} \sin (\omega t)$. Here, $A_{0}$ represents the undisturbed surface area and $a_{1}$ the amplitude of the fundamental frequency $f$. The dimensionless area amplitude $a$ is expressed as a percentage of $A_{0}: a=\left(a_{1} / A_{0}\right) \times 100(\%)$.
The dosing system for the subphase (water) is varied discretely in 3000 volume steps in arbitrary time intervals, for which a $25 \mu \mathrm{l}$ dosing syringe was used for drop volumes ranging from 5 to $13 \mu \mathrm{l}$. A fixed liquid volume per step, $0.0083 \mu \mathrm{l}$ in this case, produced a smooth sinusoidal variation of the drop area.

Inaccuracies of dosing and disturbances were quantified by the total harmonic distortion (THD) [35,36], expressed as a percentage of $a_{1}$ :

$\operatorname{THD}(\%)=\frac{\sqrt{a_{2}^{2}+a_{3}^{2}+\ldots+a_{n}^{2}}}{a_{1}} 100$

where $a_{2}, a_{3}, \ldots, a_{n}$ are amplitudes of the higher harmonics of the Fourier series.

Since ambient light affected surface tension measurements, the tensiometer was placed in a lightproof space, only illuminated by the light source (c) shown in Fig. 1.

\subsection{Tensiometer calibration}

A stainless-steel high-precision ball purchased from Martin and Co. (USA and Italy) [37] was used to calibrate the tensiometer. The ball diameter was $d=3.0 \pm 0.000125 \mathrm{~mm}$ and the deviation from the spherical form $s=0.13 \mu \mathrm{m}$. In a first calibration step, an external length scale is used, either the nozzle or the diameter of the calibration sphere. After applying the calibration software routine, the maximum deviation of the contour of the sphere image should be smaller than $5 \mu \mathrm{m}$ compared to an ideal sphere. This is achieved by properly cleaning the calibration ball and adjusting illumination and focus.

In a second step of calibration, the conversion factors from CCD-camera pixels to SI units (millimetres) in horizontal and vertical direction, $c x$ and $c z$, respectively, have to be adjusted as described in detail in [38]. A very small variation in $c x$ and/or $c z$ affects the absolute value of $\sigma$ which is checked with a calibration fluid and its literature value. An error of the $c x / c z$ ratio produces an incorrect dependence of $\sigma$ on the drop volume. Calibration is done iteratively by first varying the ratio $c x / c z$ to minimize the volume dependence of $\sigma$. Then the values of $c x$ and $c z$ are varied at constant ratio until the literature value of $\sigma$ is achieved. The surface tension values of a small drop are unreliable (e.g. for a drop area of $9.3,11.0,14.3,17.5,20.7 \mathrm{~mm}^{2}$, corresponding to a volume of $3.0,3.8,5.5,7.3,9.2 \mathrm{~mm}^{3}$, the surface tension values of pure water were $79.9,82.4,74.1,71.5,71.3$, respectively). Evidently, the drop must be large enough for gravity to clearly

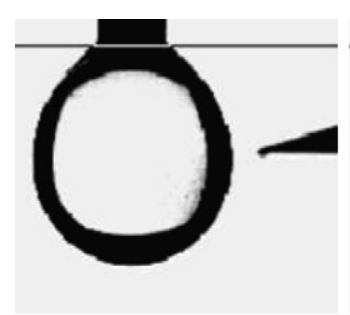

(a)

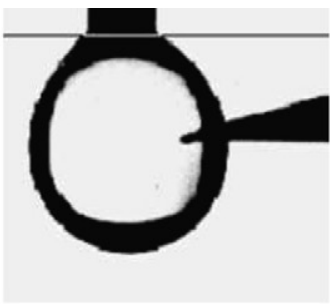

(b)

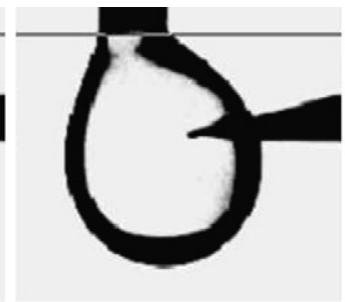

(c)

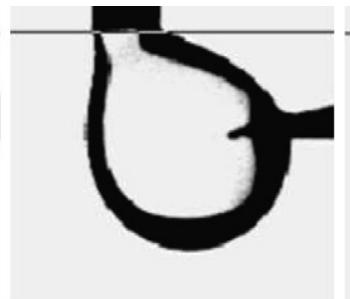

(d)

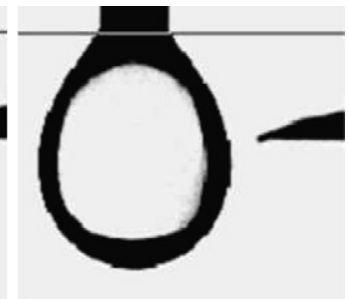

(e)

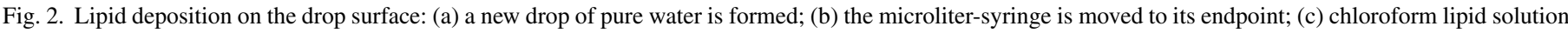
is injected at the drop surface; (d) withdrawal of microliter-syringe; (e) monolayer coated drop after evaporation of solvent. 
distort its spherical shape [39]. After calibration the drop size range for constant values of $\sigma$ is determined. For pure water the minimum drop surface area is $20 \mathrm{~mm}^{2}$ and the corresponding volume is $8.8 \mathrm{~mm}^{3}$ for the capillary diameter used $(0.7 \mathrm{~mm})$.

\subsection{Lipid deposition}

For deposition of the lipid solution on the pendent drop surface a $0.5 \mu \mathrm{l}$ Hamilton syringe with an obliquely cut tip $\left(12^{\circ}\right)$ was used. The injected volume was usually $0.2 \mu \mathrm{l}$, varying at maximum between 0.1 and $0.4 \mu \mathrm{l}$. Approximately the same volume was used by Wüstneck et al. [3]. The lipid concentration was usually $0.2 \mathrm{mM}$.

A typical injection procedure is presented in Fig. 2. A drop of water was formed (Fig. 2(a)), and the microliter-syringe moved horizontally towards the micrometer-adjustable endpoint (b). The amount of chloroform lipid solution was injected at the drop surface (c) and the microliter-syringe was slowly pulled out (d). By varying the volume of the lipid solution injected, the desired film pressure was obtained (e).

A blunt syringe tip $\left(90^{\circ}\right)$ caused vibrations upon withdrawal, which forced the drop to fall off. Therefore, only oblique syringe tips were used.

\section{Results and discussion}

\subsection{Dependence of $\eta$ and $\varepsilon$ on oscillation frequency and amplitude}

THD, $\varepsilon$ and $\eta$ of DPPC, DOPC and cholesterol monolayers as functions of $a$ and $f$ are presented in Figs. 3-5, respectively. For simplicity, the notations for THD, $\varepsilon, \eta$ and $\Pi$ in text and figures are given with the indices $\mathrm{P}, \mathrm{O}$ and $\mathrm{C}$ for DPPC, DOPC and cholesterol, respectively.

The film pressures were kept constant at $\Pi_{\mathrm{P}}=17.5 \mathrm{mN} \mathrm{m}^{-1}$ (Fig. 3), $\Pi_{\mathrm{O}}=21.7 \mathrm{mN} \mathrm{m}^{-1}$ (Fig. 4) and $\Pi_{\mathrm{C}}=16.0 \mathrm{mN} \mathrm{m}^{-1}$ (Fig. 5). The dependence on $a$ is presented in the left column, i.e. graphs $\mathrm{A}, \mathrm{C}$ and $\mathrm{E}$. The same data are analysed in their dependence on $f$ in the right column, i.e. graphs $\mathrm{B}, \mathrm{D}$ and F. The frequency $f$ was varied between 8 and $25 \mathrm{mHz}$ for all experiments. Different amplitudes $a$ were tested; for DPPC and DOPC $2.5 \% \leq a \leq 20.0 \%$; for cholesterol $1.5 \% \leq a \leq 5.0 \%$. For the cholesterol measurements, owing to its high surface elasticity (for $\Pi=16 \mathrm{mN} \mathrm{m}^{-1}$ ), higher $a$ would have forced the pendent drop to fall off when $A$ approaches the minimum value. In cases of high amplitudes the monolayer may be destroyed, which results in a breakdown of the rheological parameters [3].

The time interval for the Fourier series analysis can be chosen arbitrarily. Usually, a time interval of 4-6 periods was analysed. However, THD values depend on the interval, i.e. the number of periods and their phase. For example, an interval of 30 periods was analysed by choosing different numbers of periods and phase for the Fourier analysis (results not shown). The relative deviation of THD is $5-10 \%$, for $\eta$ it is $3.5-14 \%$ and for $\varepsilon$ it is $2.5-6.6 \%$. The fact that $\eta$ is determined with less precision was reported as well by Wüstneck et al. [2] which found the confidential intervals (95\% confidence level) of $\varepsilon$ about $1-4 \%$, whereas those for the $\eta$ between 10 and 15\%. Decesari et al. [40] obtained an uncertainty on the $\varepsilon$ determination of $10 \%$ for $\Pi>3 \mathrm{mN} \mathrm{m}^{-1}$ using the same instrument.

It was found that the highest number of periods analysed does not produce the smallest THD in general. Therefore, in Figs. 3 and 4 the results are given only for the minimum value of THD. In Figs. 5 and 6 two to five results of THD (only in Fig. 5), $\varepsilon$ and $\eta$ for the same experimental data are shown for comparison.

We will first discuss the influence of $a$ on THDvalues in the respective graphs A of Figs. 3-5. Generally, $1.7 \% \leq \mathrm{THD} \leq 19 \%$. As expected, larger amplitudes $a$ mostly cause nonlinear effects and therefore produce higher THD. However, not all curves show clear dependencies. Therefore, statistical estimations were undertaken to assess for which parameters correlations exist. A $t$-test according to Student as reported by Bronstein [41] was applied, where the distribution function $t_{p, n}$ is given by

$t_{p, n}=\frac{R}{\sqrt{1-R^{2}}} \sqrt{n-2}$

where $R^{2}$ is the coefficient of correlation of the respective (mostly linear) regression, $n$ the number of independent data, and $p$ is the probability of the hypothesis $\mathrm{H}_{0}$, where $\mathrm{H}_{0}$ means that there is no correlation.

For DPPC, as shown in Fig. 3A, THD significantly increases with $a(p<0.05)$ for four frequencies $(8,18,20,25 \mathrm{mHz})$, whereas no significant tendency $(p>0.05)$ for the remaining three frequencies $(10,12.5$ and $15 \mathrm{mHz})$ was found. Linear trendlines are shown as solid lines for $p<0.05$ and as dashed lines for $p>0.05$ in Figs. 3-5. In the case of DPPC, THD-values are extraordinarily low for the cases with $p>0.05$, i.e. $1.8 \% \leq \mathrm{THD}_{\mathrm{P}} \leq 7 \%$ for $a \geq 10 \%$. THD $\mathrm{O}$ significantly $(p<0.001)$ increases with $a$ for all $f$, and THD ${ }_{\mathrm{C}}$ increases significantly with $a$ for three $f$, whereas for the other three no tendency is observed. As shown in graphs B of Figs. 3-5, THD is not systematically influenced by $f$.

In the literature, the THD-parameter was used to quantify the inaccuracies of dosing system and disturbances $[35,36]$. We show that THD is significantly influenced by $a$, but is not influenced by $f$. Generally, a nonlinearity appears at higher $a$. Saulnier et al. [32] found that nonlinear phenomena appear for a DPPC monolayer when $a>10 \%$.

The viscosity dependence on $a$ for DPPC, DOPC and cholesterol is presented in graphs $\mathrm{C}$ of Figs. 3-5, respectively. In Fig. $3 \mathrm{C}$ it can be observed that solely for $f=8 \mathrm{mHz}, \eta_{\mathrm{P}}$ significantly decreases with $a$ from 90 to $68 \mathrm{mN} \mathrm{s} \mathrm{m}^{-1}$. For $f>8 \mathrm{mHz}$, no tendency is obtained, and $27 \mathrm{mN} \mathrm{s} \mathrm{m}^{-1} \leq \eta_{\mathrm{P}} \leq 54 \mathrm{mN} \mathrm{s} \mathrm{m}^{-1}$. For DOPC, $\eta_{\mathrm{O}}$ significantly $(p<0.05)$ increases with $a$ for three $f(10,18$, and $25 \mathrm{mHz})$, whereas no significant tendency for the remaining three frequencies $(8,15$, and $20 \mathrm{mHz})$ is found.

As can be observed from Fig. 5C, cholesterol presents a very high viscosity which highly significantly $(p<0.01)$ decreases with $a$ for all frequencies. The maximum deviation of $\eta_{\mathrm{P}}$ depending on $a$ at equal $f$ is $32 \%$, for $\eta_{\mathrm{O}}$ it is $33 \%$ and for $\eta_{\mathrm{C}}$ is $105 \%$. 

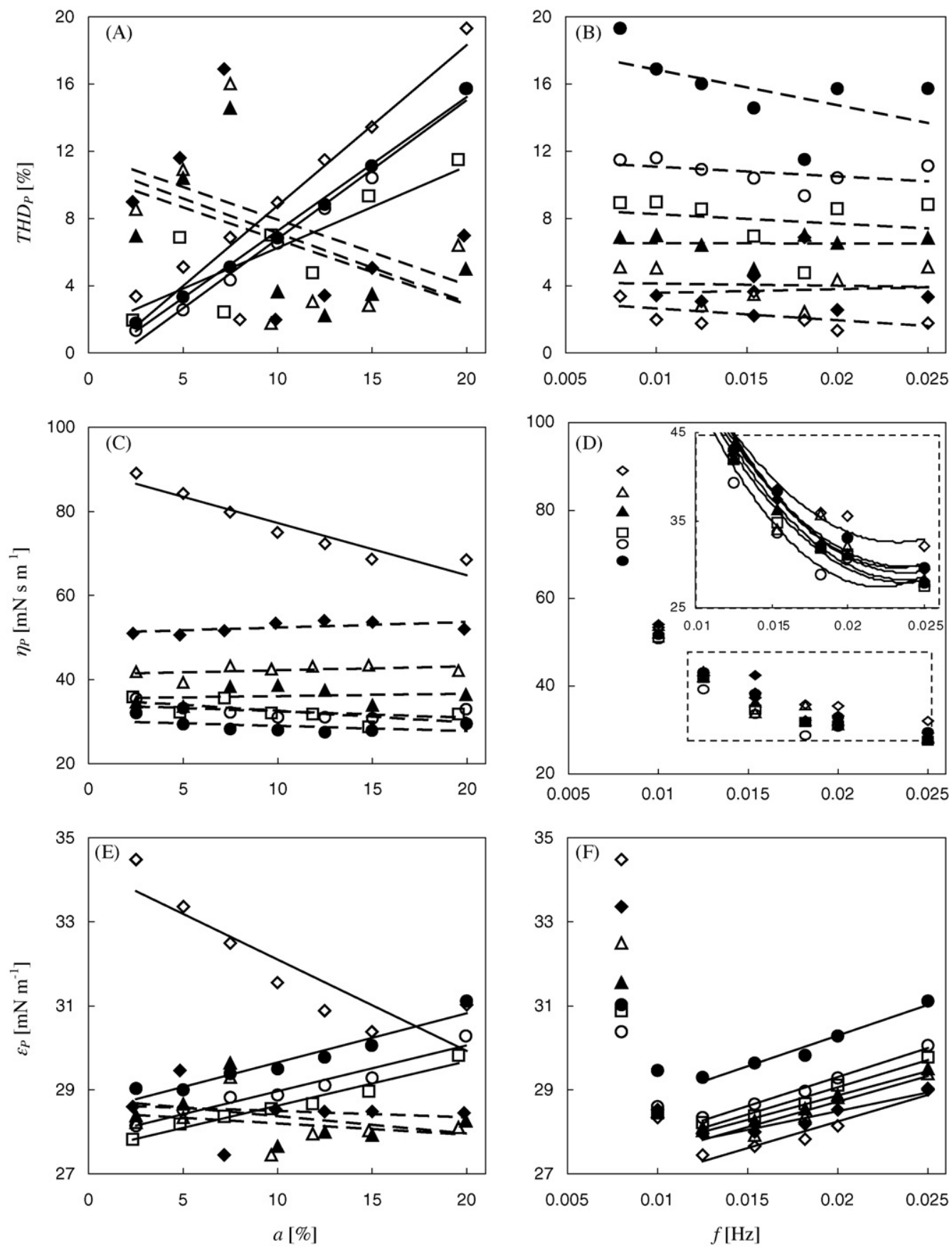

Fig. 3. DPPC monolayer at $\Pi=17.7 \mathrm{mN} \mathrm{m}^{-1}$ and $25.3^{\circ} \mathrm{C}$. (A, C and E) THDP,$\eta_{\mathrm{P}}$ and $\varepsilon_{\mathrm{P}}$ as functions of $a$ at different constant $f:(\diamond) 8 \mathrm{mHz} ;(\diamond) 10 \mathrm{mHz} ;(\triangle)$ $12.5 \mathrm{mHz} ;(\Delta) 15.4 \mathrm{mHz} ;(\square) 18 \mathrm{mHz} ;(\bigcirc) 20 \mathrm{mHz} ;(\bullet) 25 \mathrm{mHz}$. (B, D and F) THDP,$\eta_{\mathrm{P}}$ and $\varepsilon_{\mathrm{P}}$ as functions of $f$ at different constant $a:(\diamond) 2.5 \% ;(\diamond) 5.0 \% ;(\triangle)$

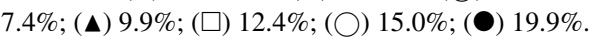

Graphs D of Figs. 3-5 present the dependence of $\eta$ on $f$. Fig. 3D contains an insertion with a zoomed area for a better distinction of data. The viscosity significantly decreases with increasing $f: p<0.025$ for DPPC, and $p<0.02$ for DOPC and cholesterol. The strong decrease of $\eta$ with increasing $f$ indicates that the surface rheological behaviour is structurally viscous, i.e. high viscosities at low frequencies, which decrease at faster deformations as already described by Wüstneck et al. [3]. Owing to its strong frequency dependence, the maximum $\eta_{\mathrm{P}}$ is $230 \%$ higher than the minimum in the range of $f$ considered, for $\eta_{\mathrm{O}}$ it is $470 \%$ and for $\eta_{\mathrm{C}}$ is $550 \%$. The viscosities of the two phospholipids, $\eta_{\mathrm{P}}$ and $\eta_{\mathrm{O}}$, are approximately in the same range $\left(\eta_{\mathrm{P}}, \eta_{\mathrm{O}}<100 \mathrm{mN} \mathrm{s} \mathrm{m}^{-1}\right)$, whereas $\eta_{\mathrm{C}}$ is much higher, i.e. $180 \mathrm{mN} \mathrm{s} \mathrm{m}^{-1}<\eta_{\mathrm{C}}<1200 \mathrm{mN} \mathrm{s} \mathrm{m}^{-1}$.

Figs. 3-5E present the variation of $\varepsilon$ with $a$. For DPPC, as shown in Fig. 3, solely for $f=8 \mathrm{mHz} \varepsilon \mathrm{P}$ significantly decreases. For low $f(10,12.5,15 \mathrm{mHz})$ there is no significant tendency $(p>0.05)$, whereas for higher $f(18,20,25 \mathrm{mHz}) \varepsilon_{\mathrm{P}}$ significantly $(p<0.05)$ increases with $a$. The maximum $\varepsilon_{\mathrm{P}}$ is $13 \%$ higher than the minimum in the measured range at equal $f$. 

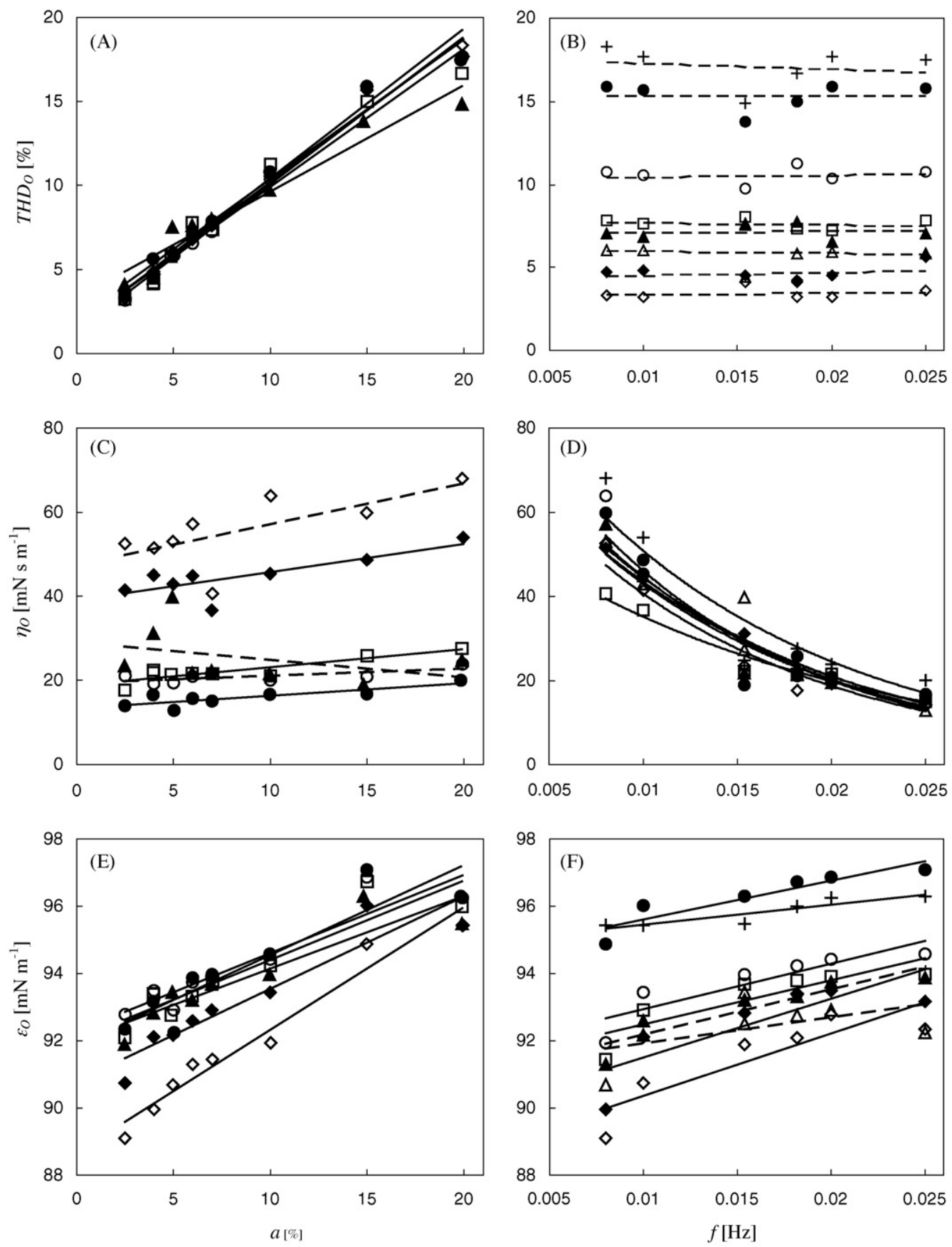

Fig. 4. DOPC monolayer at $\Pi=21.0 \mathrm{mN} \mathrm{m}^{-1}$ and $21.2^{\circ} \mathrm{C}$. (A, C and E) THD $\eta_{\mathrm{O}}$ and $\varepsilon_{\mathrm{O}}$ as functions of $a$ at different constant $f:(\diamond) 8 \mathrm{mHz} ;(\diamond) 10 \mathrm{mHz} ;(\boldsymbol{\Delta})$ $15.4 \mathrm{mHz} ;(\square) 18 \mathrm{mHz} ;(\bigcirc) 20 \mathrm{mHz} ;(\bullet) 25 \mathrm{mHz}$. (B, D and F): THD,$\eta_{\mathrm{O}}$ and $\varepsilon_{\mathrm{O}}$ as functions of $f$ at different constant $a:(\diamond) 2.5 \% ;(\diamond) 4.0 \% ;(\triangle) 5.0 \% ;(\boldsymbol{\Delta})$ $6.0 \%$; $(\square) 7.0 \%$; (○) $10.0 \%$; (๑) $15.0 \%$; (+) $20.0 \%$.

$\varepsilon_{\mathrm{O}}$ significantly $(p<0.02)$ increases with $a$ for all $f$ used. The maximum is $7 \%$ higher than the minimum in the measured range at equal $f$. Contrarily, $\varepsilon_{\mathrm{C}}$ significantly $(p<0.01)$ decreases with a. In this case, the dependency of $\varepsilon_{\mathrm{C}}$ on $f$ is diminished at higher a. The maximum $\varepsilon_{\mathrm{C}}$ is $30 \%$ higher than the minimum in the measured range at equal $f$.

Figs. 3-5F present the variation of $\varepsilon$ with $f$. In the case of DPPC, $\varepsilon_{\mathrm{P}}$ possesses a minimum at $f=12.5 \mathrm{mHz}$, whereas $\varepsilon_{\mathrm{O}}$ and $\varepsilon_{\mathrm{C}}$ are monotonically increasing with growing $f$. The maximum deviation of $\varepsilon_{\mathrm{P}}$ depending on $f$ at equal $a$ is $25 \%$, for $\varepsilon_{\mathrm{O}}$ it is $4 \%$ and for $\varepsilon_{\mathrm{C}}$ is $13 \%$.

We compare our results regarding the effect of $a$ and $f$ on $\eta$ and $\varepsilon$ as shown in Figs. 3-5 to those of other authors: Wüstneck et al. [3] also observed for DPPG that $\eta$ strongly decreases as soon as the disturbance of the monolayers is executed faster, i.e. as $f$ increases. In such cases the overall surface dilational behaviour becomes more elastic [3]. This is in agreement with our results: when $\eta$ decreases, $\varepsilon$ increases. A dependence of $\varepsilon$ 

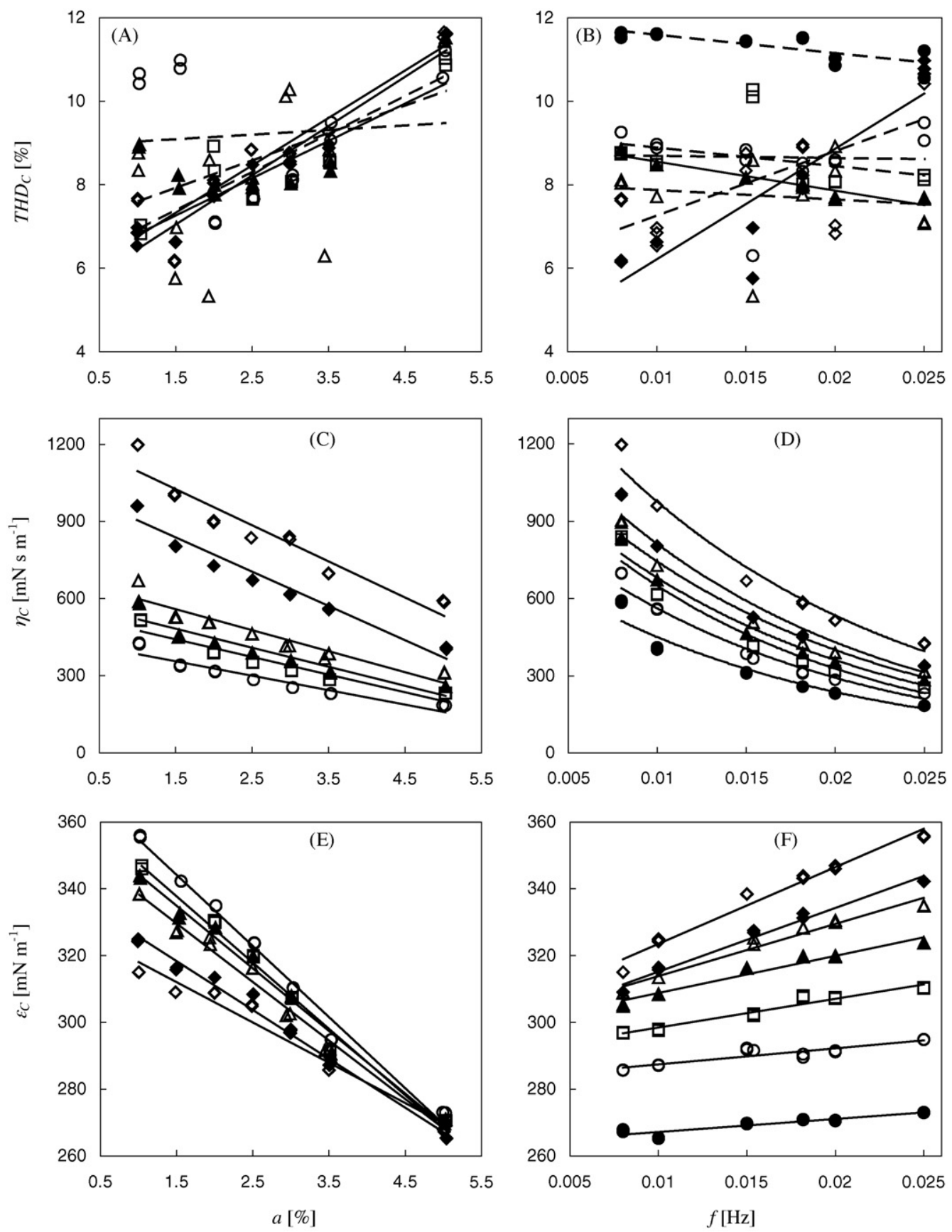

Fig. 5. Cholesterol monolayer at $\Pi=16.0 \mathrm{mN} \mathrm{m}^{-1}$ and $27.2^{\circ} \mathrm{C}$. (A, C and E) $\mathrm{THD}_{\mathrm{C}}, \eta_{\mathrm{C}}$ and $\varepsilon_{\mathrm{C}}$ as functions of $a$ at different $f:(\diamond) 8 \mathrm{mHz} ;(\diamond) 10 \mathrm{mHz} ;(\triangle) 15 \mathrm{mHz}$;

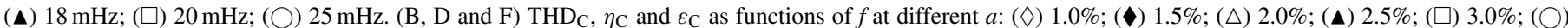
$3.5 \% ;(\bullet) 5.0 \%$.

on $f$ was reported as well by Caseli et al. [42]. Wüstneck et al. [2] studied the rheological properties of DPPC monolayers by sinusoidal oscillations of a bubble using axisymmetric bubble shape analysis. They observe that $f$ influences $\eta_{\mathrm{P}}$ and $\varepsilon_{\mathrm{P}}$ and their results are of the same order as ours. Unfortunately, a quantitative comparison is not possible because the presentation of their results is given in three-dimensional plots.

To summarize it can be stated that THD is only influenced by $a$, but not by $f$. The viscosity $\eta$ strongly decreases with increasing $f$ by a factor of 2-5. Only for cholesterol a decreasing influence with increasing $a$ can be noticed, reducing $\eta$ by a factor 2 at constant $f$. No clear influence can be seen for DPPC and DOPC, values only change by a few percent in these cases. The influence on elasticity is much smaller than on viscosity, $\varepsilon_{\mathrm{P}}$ varies by maximally $24 \%, \varepsilon_{\mathrm{O}}$ by $9 \%$ and $\varepsilon_{\mathrm{C}}$ by $34 \%$. Generally, both $a$ and $f$ influence $\varepsilon$, except for $\varepsilon_{\mathrm{P}}$, which is only increasing with $a$ for higher $f$. Mostly, $\varepsilon$ increases with increasing $a$ or $f$, only $\varepsilon_{\mathrm{C}}$ decreases with increasing $a$.

As a consequence of the results shown in Figs. 3-5, $f=18 \mathrm{mHz}$ and $a=2.5 \%$ were used to study the dependence of $\eta$ and $\varepsilon$ on $\Pi$ for pure DPPC, DOPC and cholesterol monolayers. The frequency $f$ was chosen because the dilational viscosity $\eta$ 

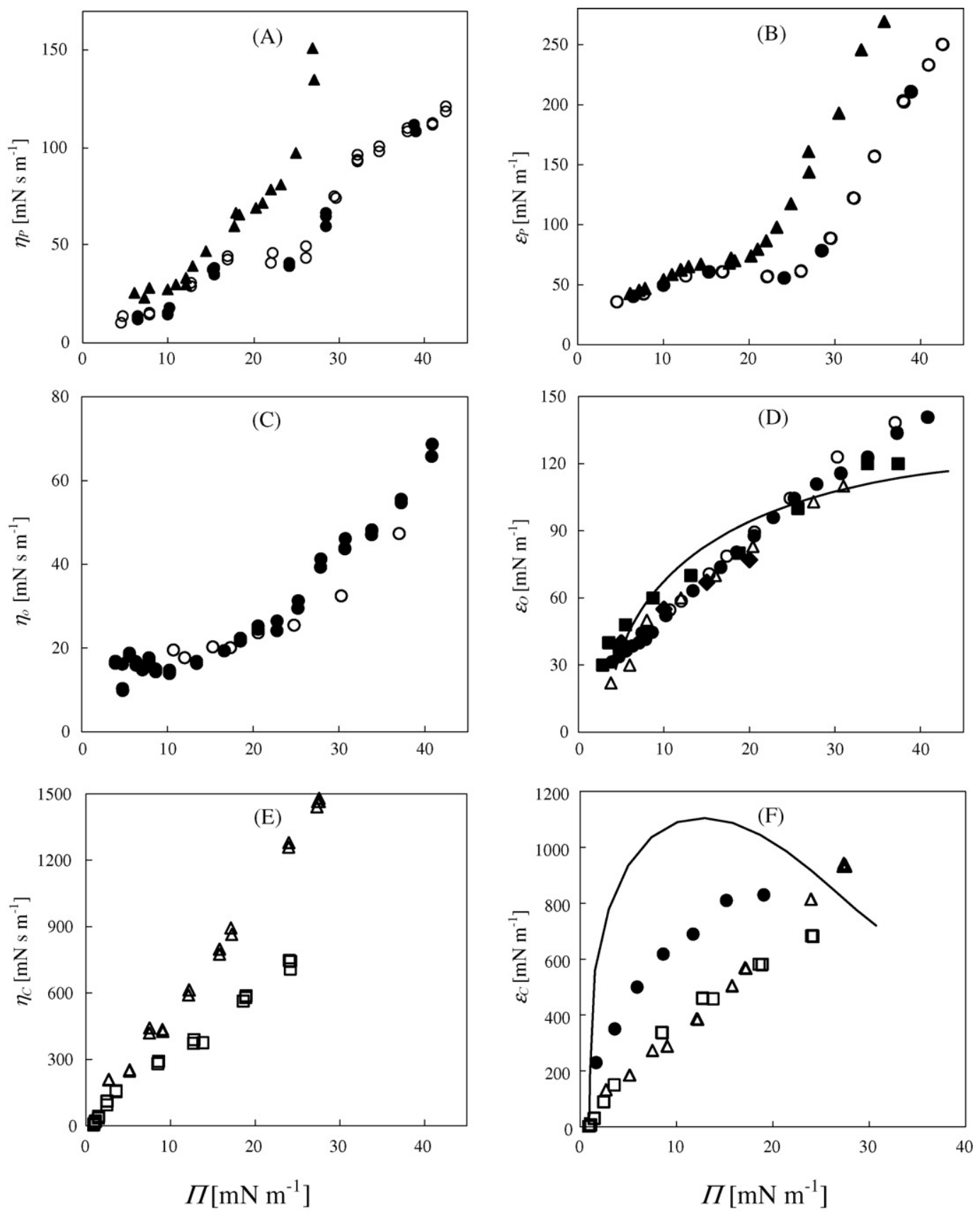

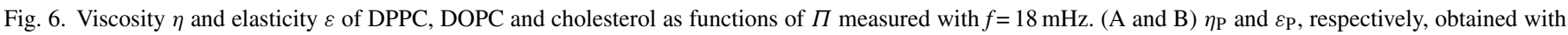

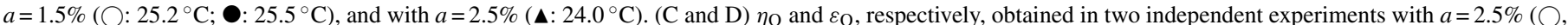

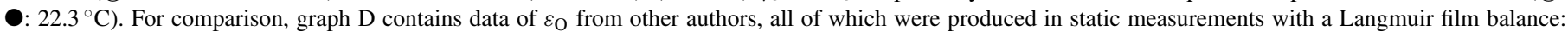

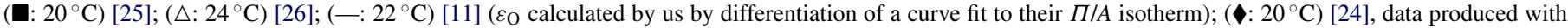

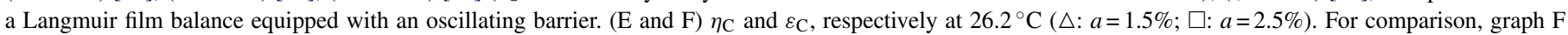

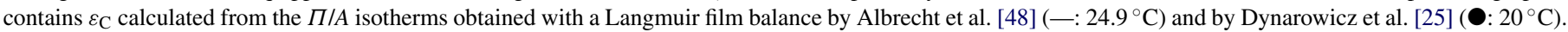

is almost constant for $f \geq 18 \mathrm{mHz}$ and it is still close to the frequency where $\varepsilon_{\mathrm{P}}$ shows a minimum. Since DPPC and cholesterol were difficult to measure at high film pressures with $a=2.5 \%$, $a=1.5 \%$ was also used in these cases.

\subsection{Dependence of $\eta$ and $\varepsilon$ on the film pressure}

Fig. 6 presents $\eta$ and $\varepsilon$ as functions of $\Pi$ for DPPC, DOPC and cholesterol. In this figure, the results were obtained with $a=1.5$ and $2.5 \%$ for DPPC (A and B) and for cholesterol (E and $\mathrm{F})$, and only with $a=2.5 \%$ for DOPC (C and D).

In graphs A-D filled and empty circles are results of two independent experiments, which are shown to prove reproducibility.

As can be observed in Fig. 6A, $\eta_{\mathrm{P}}$ generally increases monotonically, but for $a=1.5 \% \eta_{\mathrm{P}}$ presents a plateau in the range of $17 \mathrm{mN} \mathrm{m}^{-1}<\Pi<25 \mathrm{mN} \mathrm{m}^{-1}$. However, for $a=2.5 \%$ no plateau is visible. The latter curve is similar to DOPC, as $\eta_{\mathrm{O}}$ increases monotonically with $\Pi$ with no clear breaks (Fig. 6C). 


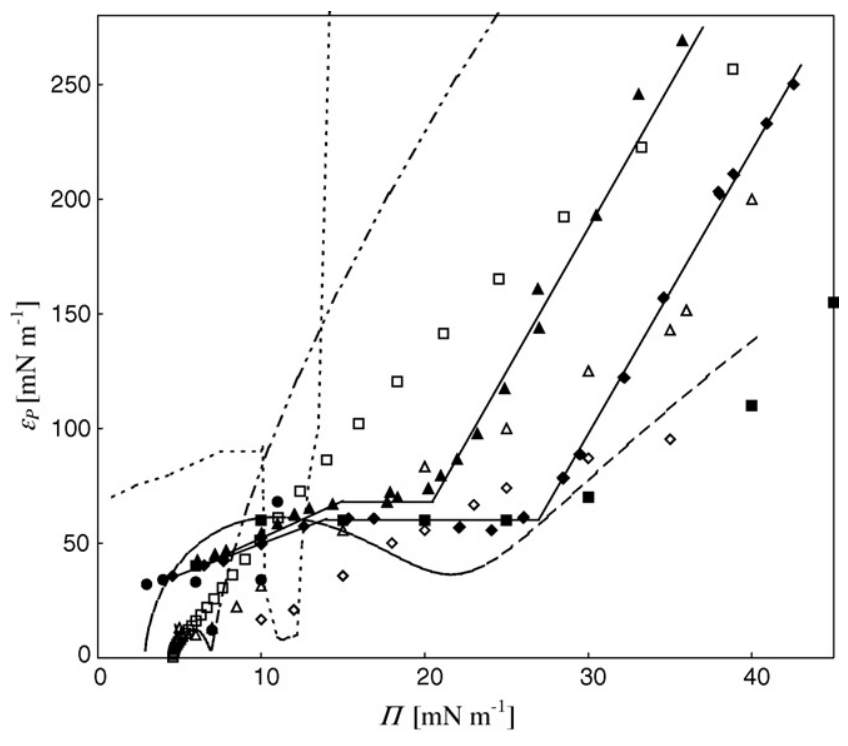

Fig. 7. DPPC elasticity measured with dynamic methods and calculated from isotherms produced in thermodynamic equilibrium. Dynamic results obtained by us with PAT $\left(\diamond: 25.2^{\circ} \mathrm{C}\right.$ and $\left.25.5^{\circ} \mathrm{C}, a=1.5 \% ; \boldsymbol{\Delta}: 24.0^{\circ} \mathrm{C}, a=2.5 \%\right)$, by Wüstneck et al. [4] with PAT (ם: $20^{\circ} \mathrm{C}$ ) and by Krägel et al. [19,20] with a Langmuir film balance equipped with an oscillating barrier $\left(20{ }^{\circ} \mathrm{C}\right) . \varepsilon_{\mathrm{P}}$ calculated from the $\Pi / A$ isotherms obtained with PAT by us $\left(---{ }_{-}: 22.4^{\circ} \mathrm{C}\right)$ and by Wüstneck et al. [4] $\left(\square: 20^{\circ} \mathrm{C},--: 30^{\circ} \mathrm{C}\right)$, and with a Langmuir film balance by Albrecht et al. [15] (- - -: $\left.24.9^{\circ} \mathrm{C}\right)$ and by Pawelec et al. [14] ( $\triangle$ : $\left.20^{\circ} \mathrm{C} ; \diamond: 25^{\circ} \mathrm{C}\right)$.

The viscosity of the saturated PC (DPPC) is always higher than that of the unsaturated PC (DOPC), i.e. $\eta_{\mathrm{P}} \geq \eta_{\mathrm{O}}$ for all $\Pi$. For $\Pi>30 \mathrm{mN} \mathrm{m}^{-1}, \eta_{\mathrm{P}}$ (obtained with $a=1.5 \%$ ) is about two times higher than $\eta_{\mathrm{O}}$.

In Fig. 6B one can see that up to $\Pi \leq 18 \mathrm{mN} \mathrm{m}^{-1}$ there is no difference between $\varepsilon_{\mathrm{P}}$ obtained with the two amplitudes, whereas for higher $\Pi$ the $\varepsilon_{\mathrm{P}}$ curves for the two amplitudes bifurcate, but still remain parallel. The plateau for $\varepsilon_{\mathrm{P}}$ with $a=2.5 \%$ is in the range of $13.5 \mathrm{mN} \mathrm{m}^{-1}<\Pi_{\mathrm{P}}<18 \mathrm{mN} \mathrm{m}^{-1}$, that for $a=1.5 \%$ is in the range of $13.5 \mathrm{mN} \mathrm{m}^{-1}<\Pi_{\mathrm{P}}<27 \mathrm{mN} \mathrm{m}^{-1}$. Apparently, the plateau of $\varepsilon_{\mathrm{P}}$ extends over a slightly larger range of the film pressure than that of $\eta_{\mathrm{P}}$ when both curves with $a=1.5 \%$ are compared. Outside the plateau, $\varepsilon_{\mathrm{P}}$ increases linearly with $\Pi$, the slope below the plateau is 4.7 times lower than that the slope above the plateau. A detailed comparison of our results of $\varepsilon_{\mathrm{P}}$ with results reported in the literature based on static and dynamic measurements will be given in Fig. 7 .

As shown in Fig. 6D for DOPC, $\eta_{\mathrm{O}}$ and $\varepsilon_{\mathrm{O}}$ increase roughly linearly with $\Pi$ without any plateau or break as in the case of $\eta_{\mathrm{P}}$ and $\varepsilon_{\mathrm{P}}$. This corresponds to the fact that DOPC presents no phase transition in its $\Pi / A$ isotherm [11,24-26,43-45]. At room temperature DOPC is in the liquid expanded state up to the point of monolayer collapse [11,45-47]. It is known that unsaturated bonds tend to create disorder in the hydrophobic region and thus hinder liquid condensed phase formation [47].

The filled symbols in Fig. 6D represent data of $\varepsilon_{\mathrm{O}}$ obtained from static measurements with a Langmuir film balance by Dynarowicz et al. [25] (filled squares) and Smaby et al. [26] (filled circles) and from dynamic measurements by Tournois et al. [24] (filled diamonds) who used a Langmuir film balance equipped with a barrier oscillating at a frequency of $0.5 \mathrm{~Hz}$. Further, $\varepsilon_{\mathrm{O}}$ was calculated from a $\Pi / A$ isotherm reported by Yan et al. [11] and is shown as solid line in Fig. 6D. As can be observed, our dynamic measurements coincide very well with the static and the dynamic results of the cited authors. We interpret the small deviation of $\varepsilon_{\mathrm{O}}$ calculated by differentiation from the $\Pi / A$ isotherm of Yan et al. [11] to be due to inevitable inaccuracies in data acquisition from isotherm curves on printed diagrams. This procedure can only produce results of limited accuracy, especially in the range of high film pressures because of the large incline of the isotherms. Since all results shown in Fig. 6D never deviate by more than $20 \%$ we conclude that $\varepsilon_{\mathrm{O}}$ curves reasonably coincide. This restriction of limited accuracy applies to all static elasticity curves which do not explicitly present $\varepsilon$, so that $\varepsilon$ had to be derived by us from their $\Pi / A$-isotherms, and which will be shown below.

Fig. $6 \mathrm{E}$ and $\mathrm{F}$ present $\eta_{\mathrm{C}}$ and $\varepsilon_{\mathrm{C}}$ obtained at $a=1.5$ and $2.5 \%$, respectively. One can observe a steep, linear increase of both $\eta_{\mathrm{C}}$ and $\varepsilon_{\mathrm{C}}$ with $\Pi$ without a plateau. There is a strong dependence of $\eta_{\mathrm{C}}$ on $a$, whereas no clear dependence of $\varepsilon_{\mathrm{C}}$ on $a$ can be observed. The values of $\eta_{\mathrm{C}}$ obtained with $a=1.5 \%$ are larger as those obtained with $a=2.5 \%$ for $\Pi \geq 10 \mathrm{mN} \mathrm{m}^{-1}$, in agreement with Fig. 5 where this was shown solely for $\Pi=16.0 \mathrm{mN} \mathrm{m}^{-1}$. For comparison, $\varepsilon_{\mathrm{C}}$ was read from a $\varepsilon_{\mathrm{C}} / A$ diagram by Dynarowicz et al. [25] (filled circles) which calculated $\varepsilon_{\mathrm{C}}$ by differentiation from their $\Pi / A$ isotherm obtained with a Langmuir film balance. The solid line in Fig. $6 \mathrm{~F}$ presents $\varepsilon_{\mathrm{C}}$ calculated by us from a $\Pi / A$ isotherm reported by Albrecht et al. [48]. Here, the static values of $\varepsilon_{\mathrm{C}}$ clearly deviate from our dynamic results.

The isotherm of cholesterol shows a single phase with a collapse pressure of approximately $45 \mathrm{mN} \mathrm{m}^{-1}$, at which point the molecular area is $\sim 39 \AA^{2}[15,46,49]$. It remains in the solid condensed phase up to the point of monolayer collapse [46]. As can be observed from Fig. 6, the cholesterol elasticity and viscosity are approximately 10 times higher than those of DPPC and DOPC.

Fig. 7 shows a comparison of our data with literature values of $\varepsilon_{\mathrm{P}}$ measured both with dynamic methods and derived from $\Pi / A$ isotherms which are assumed to be in thermodynamic equilibrium. Moreover, results obtained by two different instruments are compared in Fig. 7: profile analysis tensiometry (PAT) and the Langmuir film balance technique.

First, we will discuss $\varepsilon_{\mathrm{P}}$ values obtained with dynamic methods. Linear regressions of our $\varepsilon_{\mathrm{P}}$ values produced by harmonic oscillations are shown as solid lines. Up to $\Pi \leq 27 \mathrm{mN} \mathrm{m}^{-1}$ $\varepsilon \mathrm{P}$ values obtained by us with $a=1.5 \%$ are in very good concordance with those of Wüstneck et al. [4], who performed monolayer stepwise compressions with a pendent drop tensiometer, as described in [50]. For $\Pi>27 \mathrm{mN} \mathrm{m}^{-1}$, which limits the plateau of $\varepsilon_{\mathrm{P}}$, their curve increases with a smaller slope. Additionally, they observed brittle monolayer structures and ruptures for $\Pi>25 \mathrm{mN} \mathrm{m}^{-1}$. Further, our results are in reasonable agreement with results obtained using a modified Langmuir through with an oscillating barrier by Krägel et al. [19,20].

Focussing now on the static elasticity values calculated from $\Pi / A$ isotherms, it can be seen that they are of the same order of magnitude and have roughly the same tendency as the values 


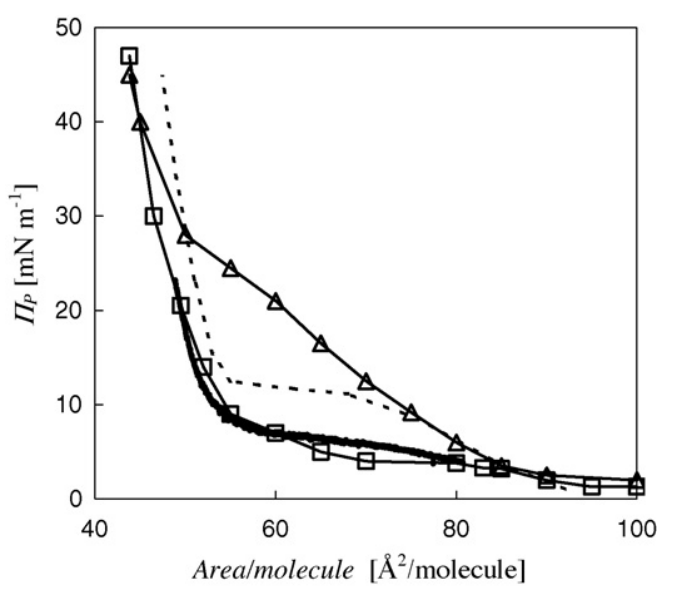

Fig. 8. DPPC isotherm measured statically with PAT by us (-: $\left.22.4^{\circ} \mathrm{C}\right)$ in comparison with results obtained by Wüstneck et al. [4] with PAT ( $\square: 20^{\circ} \mathrm{C}, \triangle$ : $\left.30^{\circ} \mathrm{C}\right)$ and by Albrecht et al. [15] with a Langmuir film balance (- - -: $\left.24.9^{\circ} \mathrm{C}\right)$.

obtained with dynamic methods. In order to discuss the static $\varepsilon_{\mathrm{P}}$ curves in Fig. 7, first the $\Pi / A$ isotherms of DPPC shown in Fig. 8 have to be introduced. They were produced by both PAT and Langmuir film balance measurements by different authors. Our isotherm obtained at $22.4{ }^{\circ} \mathrm{C}$ measured statically with PAT is in very good neighbourhood with that obtained by Wüstneck et al. [4] at $20^{\circ} \mathrm{C}$ (also measured with PAT). The former has its plateau around $6 \mathrm{mN} \mathrm{m}^{-1}$ (for $22.4^{\circ} \mathrm{C}$ ), the latter around $4 \mathrm{mN} \mathrm{m}^{-1}$ (for $20{ }^{\circ} \mathrm{C}$ ).

The isotherm obtained with a Langmuir film balance at $24.9^{\circ} \mathrm{C}$ by Albrecht et al. [15] presents a plateau around $11 \mathrm{mN} \mathrm{m}^{-1}$. For static measurements with PAT [4], a plateau no longer exists at $30^{\circ} \mathrm{C}$. At higher temperatures, the plateau is replaced by a straight incline (see Figs. 3 and 4 of [4]), which corresponds to a constant $\varepsilon_{\mathrm{P}}$ in that range. As can be seen in Fig. 8, the isotherms are strongly influenced by temperature, but all match qualitatively. Differences caused by the two different methods (PAT versus Langmuir film balance) cannot be detected here due to their differences in temperature. However, it has been shown by Wüstneck et al. [4] that both methods come to almost identical results under optimised spreading conditions, but nonoptimised spreading conditions can strongly distort the results of PAT measurements.

Moving back to Fig. 7 it can be observed that the elasticity curves calculated from the different $\Pi / A$ isotherms largely diversify. For those $\Pi / A$ isotherms with a plateau, indicating a transition from the liquid expanded (LE) to the liquid condensed (LC) state, consequently a minimum of the elasticity curve occurs. This can be observed for $\varepsilon_{\mathrm{P}}$ calculated from our $\Pi / A$ isotherm curves obtained with PAT at $22.4{ }^{\circ} \mathrm{C}$ (dash-doubledotted line in Fig. 7). The same can be observed for $\varepsilon_{\mathrm{P}}$ calculated from isotherms by Albrecht et al. [15] obtained with a Langmuir film balance at $24.9{ }^{\circ} \mathrm{C}$ (short dashed line). Our $\varepsilon_{\mathrm{P}}$ minimum is at $\Pi=7 \mathrm{mN} \mathrm{m}^{-1}$, whereas $\varepsilon_{\mathrm{P}}$ calculated from Albrecht et al. [15] is at $11 \mathrm{mN} \mathrm{m}^{-1}<\Pi<12 \mathrm{mN} \mathrm{m}^{-1}$. $\varepsilon_{\mathrm{P}}$ calculated from an isotherm obtained with PAT at $20^{\circ} \mathrm{C}$ by Wüstneck et al. [4] monotonically increases with $\Pi$ (open squares) whereas the one at $30{ }^{\circ} \mathrm{C}$ presents a minimum at $\Pi=22 \mathrm{mN} \mathrm{m}^{-1}$ (long dashed line). $\varepsilon_{\mathrm{P}}$ calculated from isotherms obtained by Pawelec et al. [14] at two different temperatures monotonically increase (open triangles and diamonds).

With increasing temperature the dilational elasticity calculated from isotherms decreases. This can be observed from the two data sets at different temperatures produced with PAT by Wüstneck et al. [4] and with a Langmuir film balance by Pawelec et al. [14].

Dynamic methods show a plateau of an approximately constant elasticity value for a certain interval of $\Pi$. Static (equilibrium) methods show a minimum of elasticity when there is a plateau in the $\Pi / A$ isotherms, elsewhere a monotonic increase of elasticity is obtained. Thus, the discrepancies of the surface elasticities of DPPC mainly result from the dynamic or static conditions of the measurement, while in thermodynamic equilibrium good agreement is between PAT and Langmuir film balance results for $\Pi / A$ isotherms is documented in Fig. 8.

\section{Discussion}

The frequency dependence of the dilational viscosity $\eta$ and elasticity $\varepsilon$ for both phospholipids and cholesterol is a clear indication of specific time scales for the reorganization processes in the monolayers. Based on stress-relaxation experiments, Joos et al. [51] approximated the decay of film pressure in time and its approach to an equilibrium value after a stepwise compression with two independent frequency constants $k_{1}$ and $k_{2}$ :

$\Pi(t)=\Pi(t=0)\left\{\beta \exp \left(-k_{1} t\right)+(1-\beta) \exp \left(-k_{2} t\right)\right\}$

with $0 \leq \beta \leq 1$

Although their values obtained for DPPC and cholesterol scatter widely, they still allow to estimate the orders of magnitude of the relaxation process: for DPPC, $k_{1}=13.0 \pm 6.1 \mathrm{mHz}$ (average \pm standard deviation), $k_{2}=0.46 \pm 0.24 \mathrm{mHz}$ with the partition factor $\beta=0.25 \pm 0.09$. Both $k_{1}$ and $k_{2}$ are not significantly correlated with $\Pi$ in the full measured range $3 \mathrm{mN} \mathrm{m}^{-1} \leq \Pi \leq 25 \mathrm{mN} \mathrm{m}^{-1}(p>0.1)$. Approximating stress relaxation with a single constant, Wüstneck et al. [4] found the frequency constant to be about $16 \mathrm{mHz}$ for compression and about $18 \mathrm{mHz}$ for expansion in a wide range of specific area $A\left(45 \AA^{2} \leq A \leq 65 \AA^{2}\right.$ corresponding to $\left.5 \mathrm{mN} \mathrm{m}^{-1} \leq \Pi \leq 30 \mathrm{mN} \mathrm{m}^{-1}\right)$. Only for the highest film pressures $\left(\Pi \approx 47 \mathrm{mN} \mathrm{m}^{-1}\right)$ they found the constant to be about $14 \mathrm{mHz}$ for both compression and expansion.

The frequency constant $k_{1}$ and the constants reported by Wüstneck et al. [4] coincide well with the minimum of $\varepsilon_{\mathrm{P}}$ found by us at $12.5 \mathrm{mHz}$. According to the generalized Maxwell model of viscoelasticity (also known as Maxwell-Weichert model) relaxation can be compared to a parallel arrangement of one elastic spring and several pairs of one elastic spring (with elastic modulus $\varepsilon_{i}$ ) coupled with a viscous dashpot (with viscosity $\eta_{i}$ ) in series. Each spring/dashpot pair has its own relaxation time constant $\tau_{i}=\eta_{i} / \varepsilon_{i}(i=1, \ldots, n)$ which contributes to the relaxation time distribution of the whole system. For oscillations with a period $t<\tau_{i}$ the respective spring/dashpot pair contributes more 
to the elastic response, for $t>\tau_{i}$ it contributes more to the viscous response. Therefore, we interpret the coincidence of the minimum of $\varepsilon$ P with the relaxation frequency reported by Joos et al. [51] and Wüstneck et al. [4] in the following manner: if harmonic oscillations are produced to measure $\varepsilon_{\mathrm{P}}$ in the range of the relaxation frequency $k_{1}$, the elastic response is minimized.

The question remains why $\varepsilon$ P increases for $f<12.5 \mathrm{mHz}$ ? According to Joos et al. [51] a second relaxation time constant $k_{2}=0.46 \pm 0.24 \mathrm{mHz}$ has to be taken into account which contributes much stronger to the relaxation process than $k_{1}$ owing to $\beta=0.25 \pm 0.09$. Unfortunately, we could not find any similar results in the literature reporting about a local minimum of the elasticity of a viscoelastic material from either theoretical or experimental studies. Therefore, we can only speculate that, speaking in terms of the Maxwell model, a coupling effect of the different spring elastic moduli $\left(\varepsilon_{i}\right)$ may occur for oscillation periods smaller than $\tau_{1}$ resulting in an increase of the total elasticity of the system for a certain band of frequencies. Coupling of different factors contributing to molecular relaxation could then be the cause for a local increase of the apparent elasticity of the monolayer with decreasing $f$. However, an extension of the frequency range well below $k_{2}$ would be necessary in future experiments to set a sound basis for an interpretation of this effect. For cholesterol, [51] report $k_{1}=538 \pm 268 \mathrm{mHz}$, $k_{2}=40.7 \pm 26.7 \mathrm{mHz}$ with the partition factor $\beta=0.27 \pm 0.30$. In our measurements $\varepsilon_{\mathrm{C}}$ shows a slow monotonic increase of $14 \%$ (for the smallest amplitude $a=1 \%$ ) with increasing $f$ over the range considered $(8 \mathrm{mHz} \leq f \leq 25 \mathrm{mHz})$. We interpret the fact that we do not find a minimum here to be a consequence of the frequency interval of our measurements being lower than the mentioned relaxation frequencies $k_{1}$ and $k_{2}$. This would mean that the cholesterol monolayer has enough time to reorient completely within a drop oscillation cycle.

As we did not find any stress relaxation measurements for DOPC or other unsaturated phospholipids, there is no sound basis to interpret the frequency dependence of $\varepsilon_{\mathrm{O}}$ so far.

We will now consider the frequency dependence of the dilational viscosity $\eta$. A strong decrease of surface dilational viscosity with increasing $f$ is also reported by Wüstneck et al. [3] for DPPG. Wüstneck et al. [3] interpret this fact in analogy to the "shear thinning" properties of some bulk liquids as "structural viscosity". But unlike Wüstneck et al. [3], who find a local minimum of $\eta$ for DPPG around $18 \mathrm{mHz}$ and an increase of $\eta$ at higher $f$, we do not find a minimum for DPPC, DOPC and cholesterol in the measured range. Instead, our dilational viscosity curves seem to approach a minimum at higher $f$. We interpret these facts in the sense that dilational viscosity is higher the more the monolayer approaches a relaxed state and is diminished when the monolayer is in a stressed or unrelaxed state which is well in accordance with the generalized Maxwell model stated above. Since viscosity is diffusion of momentum by molecular interaction, this means that interactions are stronger in the relaxed than in the unrelaxed (stressed) state.

This conclusion can also be applied to the amplitude dependence of $\eta$, see graphs $\mathrm{C}$ of Figs. 3-5. As shown in Fig. 5D for cholesterol, $\eta_{\mathrm{C}}$ is largest at the lowest amplitude $a=1.0 \%$ and is reduced to $49 \%$ of its maximum value when $a$ is increased to $5 \%$.
This shows that a cholesterol monolayer at $\Pi_{\mathrm{C}}=16.0 \mathrm{mN} \mathrm{m}^{-1}$ is very sensitive to area variations. It is immediately transferred into a stressed state, and consequently a decrease of molecular interactions occurs. For DPPC, an amplitude dependence of $\eta_{\mathrm{P}}$ only occurs at the lowest frequency $f=8 \mathrm{mHz}$, for all other frequencies $\eta_{\mathrm{P}}$ is not altered by $a$. This means that only for $f=8 \mathrm{mHz}$ a DPPC monolayer is sufficiently relaxed to be sensitive for the stressing effect of an increased oscillation amplitude. Since amplitude effects are not systematic for $\eta_{\mathrm{O}}$, we will not interpret them.

Considering amplitude effects of surface elasticity $\varepsilon$, we can see that for cholesterol $\varepsilon_{\mathrm{C}}$ decreases with increasing $a$ for all $f$, whereas for DOPC $\varepsilon_{\mathrm{O}}$ increases with increasing $a$ for all $f$. A mixed case occurs for DPPC: here, $\varepsilon_{\mathrm{P}}$ decreases with increasing $a$ for $f=8 \mathrm{mHz}$, it remains indifferent in the range $10 \mathrm{mHz} \leq f \leq 15 \mathrm{mHz}$ and increases with increasing $a$ for $f \geq 18 \mathrm{mHz}$. This means that below the relaxation frequency $f \approx 12.5 \mathrm{mHz}$ DPPC has the same amplitude dependence as cholesterol and above this relaxation frequency it adopts that of DOPC. As stated above, the cholesterol monolayer is assumed to be in an (almost) relaxed state in the range of frequencies studied which are all smaller than the two relaxation frequencies $k_{1}$ and $k_{2}$. We try to interpret this effect making use again of the generalized Maxwell model of viscoelasticity which is composed of several elastic springs and viscous dashpots. The elastic potential energy $E$ of each spring $i$ is given by $E_{i}=\varepsilon_{i} x^{2} /(2 l)$, where $x$ is the strain and $l$ is the length of the spring. Since larger drop oscillation amplitudes $a$ corresponding to larger spring strains $x$ apparently decrease the total elasticity $\varepsilon$ of the monolayer, the elastic potential energy of the monolayer increases less than with the factor $a^{2}$. This means that less elastic potential energy is conserved in a monolayer than one would expect from linear stress/strain response corresponding to a constant $\varepsilon$. Thus, the change of $\varepsilon$ with increasing $a$ has to be regarded as a nonlinear effect. Since the cholesterol monolayer is in an ordered, solid-like state, higher amplitudes seem to decrease the degree of order and thus the possibility to conserve elastic potential energy. Contrary to cholesterol, DOPC is in a low order liquid expanded state in the whole film pressure range. Here, higher amplitudes apparently increase the amount of elastic potential energy conservable in the monolayer, presumably accompanied by a slight increase of order. In the case of DPPC it seems that the relaxation frequency $k_{1}$ is the critical parameter: for oscillation frequencies $f<k_{1}$ where the first step of the relaxation process is assumed to be completed, higher amplitudes decrease $\varepsilon$ p, presumably accompanied by a decrease in order. For oscillation frequencies $f>k_{1}$ higher amplitudes increase $\varepsilon \mathrm{P}$, presumably accompanied by an increase in order. It appears worth noting that also the $\mathrm{THD}_{\mathrm{P}}$ dependence on amplitude $a$ is sensitive on the relaxation frequency $f \approx 12.5 \mathrm{mHz}$, since for $f=10,12.5,15.4 \mathrm{mHz}$, THDP does not increase with $a$ and shows very low THDP-values. Outside this frequency range $(f=8,18,20,25 \mathrm{mHz})$, THD increase with increasing $a$.

How could a relaxed and an unrelaxed state differ in their internal structure? As will be discussed below in more detail for DPPC, this monolayer is in a coexistence state of liquid crystalline, micron sized domains surrounded by a fluid monolayer 
phase in the liquid expanded state [16] at $\Pi_{\mathrm{P}}=17.5 \mathrm{mN} \mathrm{m}^{-1}$ as used for Fig. 3. The compression and expansion of the film through a harmonic area change alters the size and shape of these domains. It is shown that faster compression/expansion rates result in smaller domain sizes [45]. Cycling the monolayer several times between low and high pressures is reported to yield a "snowstorm" pattern-a large number of small domains of similar size and shape [44]. In contrast, cholesterol is in a liquid crystalline state [15] at $\Pi_{\mathrm{C}}=16.0 \mathrm{mN} \mathrm{m}^{-1}$ (as used for Fig. 5), whereas DOPC is in the liquid expanded state at $\Pi_{\mathrm{O}}=21.7 \mathrm{mN} \mathrm{m}^{-1}$ (as used for Fig. 4). For the latter two cases no domain formation is reported. This has to be taken into account for the relaxation processes discussed above.

We will now discuss the dependence of $\eta$ and $\varepsilon$ on the film pressure $\Pi$. Focusing first on $\varepsilon \mathrm{P}$ we can see its plateau in the range $13.5 \mathrm{mN} \mathrm{m}^{-1} \leq \Pi \leq 27 \mathrm{mN} \mathrm{m}^{-1}$ (for $a=1.5 \%$ ) coincides reasonably well with phase transitions reported from isotherm measurements by Albrecht et al. [15] at 12 and $25 \mathrm{mN} \mathrm{m}^{-1}$. The first phase transition at $\Pi=12 \mathrm{mN} \mathrm{m}^{-1}$ is described by Albrecht et al. [15] as the "liquidus line separating phases containing fluid DPPC from phases with crystalline lecithin". Albrecht et al. do not comment on the second phase transition explicitly, but it can be deduced unambiguously from their compressibility data (their Fig. 4). As is described for many isotherms, e.g. for DMPE (see [52]), the kink in the isotherm in the film pressure range of $25-30 \mathrm{mN} \mathrm{m}^{-1}$ is referred to as the transition between a coexistence region and a homogeneous solid phase. We therefore conclude that in dynamic surface elasticity measurements $\varepsilon_{\mathrm{P}}$ is constant over the full range of the coexistence region of crystalline DPPC domains embedded in fluid monolayer phase. Contrary to that, $\Pi / A$-isotherms measured in equilibrium exhibit a plateau in $\Pi$ only at the lower bound of the coexistence region.

Why do the two $\varepsilon_{\mathrm{P}}$ curves produced with $a=1.5$ or $2.5 \%$ deviate for film pressures $\Pi>18 \mathrm{mN} \mathrm{m}^{-1}$ ? Taking into account the $\Pi / A$-isotherm shown in Fig. 8 it can be seen that when the area $A$ is reduced by $2.5 \%$ at a film pressure $\Pi=18 \mathrm{mN} \mathrm{m}^{-1}$ this compression produces a film pressure $\Pi=23 \mathrm{mN} \mathrm{m}^{-1}$. This means that during each compression cycle a phase transition to the solid state occurs for film pressures $\Pi>18 \mathrm{mN} \mathrm{m}^{-1}$ when $a=2.5 \%$ is used. Thus, owing to the steep increase in $\Pi$ at low $A$, only small oscillation amplitudes $a$ should be used in these cases. We therefore exclude the $\eta_{\mathrm{P}}$ and $\varepsilon_{\mathrm{P}}$ curves produced with $a=2.5 \%$ from further consideration in the range $\Pi>18 \mathrm{mN} \mathrm{m}^{-1}$.

\section{Conclusion}

Testing the influences of the amplitude $a$ and frequency $f$ of the forced harmonic area oscillations on the total harmonic distortion (THD) it could be shown that $f$ does not influence the THD, whereas the THD increases with increasing $a$ in many, but not all cases.

The frequency $f$ clearly influences both the surface elasticity $\varepsilon$ and the dilational viscosity $\eta$. For DPPC it could be shown that $\varepsilon_{\mathrm{P}}$ possesses a minimum at $f=12.5 \mathrm{mHz}$. This coincides well with the relaxation frequencies $k$ measured in stress relaxation experiments by Joos et al. [51] with their first relaxation frequency $k_{1}=13.0 \pm 6.1 \mathrm{mHz}$ and by Wüstneck et al. [4] who found $14 \mathrm{mHz} \leq k \leq 18 \mathrm{mHz}$. The generalized Maxwell model can be used to interpret this coincidence in the way that around the relaxation frequency the elasticity is minimized.

The viscosity $\eta$ decreases with increasing $f$ for all monolayers tested by a factor of 2-5, i.e. for faster compression/expansion rates dilational viscosity is strongly reduced and appears to approach a minimum outside the measured range. A strong decrease of surface dilational viscosity with increasing $f$ is also reported by Wüstneck et al. [3] for DPPG. We conclude that dilational viscosity is higher the more the monolayer approaches a relaxed state and is diminished when the monolayer is in a stressed or unrelaxed state. Thus, the molecular interactions are stronger in the relaxed than in the stressed state.

Comparing dynamic influences on surface elasticity $\varepsilon$ and dilational viscosity $\eta$ it can be concluded that $\varepsilon$ is influenced by the dynamic parameters to a much smaller extent than $\eta$. The surface elasticity $\varepsilon$ varies by maximally $24 \%$ for DPPC, by $9 \%$ for DOPC and by $34 \%$ for cholesterol in the range of parameters considered.

Good reproducibility of $\varepsilon$ and $\eta$ produced with PAT measurements is shown over the entire range of the film pressure $\Pi$, but the accuracy in the determination of $\varepsilon$ is higher than for $\eta$. The surface dilational viscosity of the saturated phospholipid DPPC is higher than that of the unsaturated phospholipid DOPC for all $\Pi$, whereas $\varepsilon$ and $\eta$ of cholesterol are about ten times higher than DPPC and DOPC.

The dynamic surface elasticity $\varepsilon_{\mathrm{P}}$ of DPPC exhibits a plateau in the range $13.5 \mathrm{mN} \mathrm{m}^{-1} \leq \Pi \leq 27 \mathrm{mN} \mathrm{m}^{-1}$ which coincides with phase transitions reported for (static) isotherm measurements by Albrecht et al. [15] at 12 and $25 \mathrm{mN} \mathrm{m}^{-1}$. These phase transitions are regarded as the lower and upper bound of the so-called coexistence region of micron sized liquid crystalline domains surrounded by a fluid monolayer phase. We therefore conclude that in dynamic surface elasticity measurements $\varepsilon_{\mathrm{P}}$ is constant over the full range of this coexistence region whereas in equilibrium measurements ( $\Pi / A$-isotherms) a plateau in $\Pi$ is only seen at the lower bound of the coexistence region. The plateau described here for $\varepsilon_{\mathrm{P}}$ is quantitatively confirmed by dynamic measurements of [4]. For DOPC and cholesterol no plateau is found for $\eta$ and $\varepsilon$.

Very good agreement between dynamic results of $\varepsilon_{\mathrm{O}}$ and those derived from an isotherm (static $\varepsilon_{O}$ ) is found for the full range of $\Pi$ when measured with two different methods (PAT and Langmuir film balance).

As proved by Wüstneck et al. [4] and confirmed in this study, $\Pi / A$ isotherms of DPPC produced by either PAT or a Langmuir film balance closely coincide, leading to comparable static surface elasticities at equal temperatures, which were deduced from the isotherm measurements.

Static and dynamic results of the elasticities of DPPC and cholesterol strongly deviate. Elasticities measured statically are higher than those measured dynamically at equal temperatures.

\section{Acknowledgments}

It is a pleasure for us to express our gratitude to Dr. R. Miller, MPI Golm, and to Dr. A. Makievski, Sinterface, Berlin, 
for their scientific advices concerning measurement accuracies and inspiring discussions. We are indebted to the reviewers for their competent criticisms and helpful advises. We thankfully acknowledge technical assistance rendered by S. Nikolaus and P. Wandrés, and funding by the Deutsche Forschungsgemeinschaft (DFG), LE 1119/3-1.

\section{References}

[1] H. Hamm, H. Fabel, W. Bartsch, The surfactant system of the adult lung: physiology and clinical perspectives, Clin. Invest. 70 (1992) 637-657.

[2] N. Wüstneck, R. Wüstneck, V.B. Fainerman, R. Miller, U. Pison, Interfacial behaviour and mechanical properties of spread lung surfactant protein/lipid layers, Colloids Surf. B 21 (2001) 191-205.

[3] R. Wüstneck, P. Enders, N. Wüstneck, U. Pison, R. Miller, D. Vollhardt, Surface dilational behaviour of spread dipalmitoyl phosphatidyl glycerol monolayers, Phys. Chem. Commun. 11 (1999).

[4] R. Wüstneck, N. Wüstneck, D.O. Grigoriev, U. Pison, R. Miller, Stress relaxation behaviour of dipalmitoyl phosphatidylcholine monolayers spread on the surface of a pendant drop, Colloids Surf. B 15 (1999) 275-288.

[5] D.O. Grigoriev, J. Kragel, A.V. Akentiev, B.A. Noskov, R. Miller, U. Pison, Relation between rheological properties and structural changes in monolayers of model lung surfactant under compression, Biophys. Chem. 104 (2003) 633-642.

[6] A. Fillery-Travis, L.H. Foster, M.M. Robins, Stability of emulsions stabilized by two physiological surfactants: L-alpha-phosphatidylcholine and sodium taurocholate, Biophys. Chem. 54 (1995) 253-260.

[7] X. Li, C. Pozrikidis, The effect of surfactants on drop deformation and on the rheology of dilute emulsions in Stokes flow, J. Fluid Mech. 341 (1997) 165-194.

[8] S.L. Veatch, I.V. Polozov, K. Gawrisch, S.L. Keller, Liquid domains in vesicles investigated by NMR and fluorescence microscopy, Biophys. J. 86 (2004) 2910-2922.

[9] T.J. McIntosh, A. Vidal, S.A. Simon, Sorting of lipids and transmembrane peptides between detergent-soluble bilayers and detergent-resistant rafts, Biophys. J. 85 (2003) 1656-1666.

[10] S.L. Veatch, S.L. Keller, Organization in lipid membranes containing cholesterol, Phys. Rev. Lett. 89 (2002) 2681011-2681014.

[11] W. Yan, B. Piknova, S.B. Hall, The collapse of monolayers containing pulmonary surfactant phospholipids is kinetically determined, Biophys. J. 89 (2005) 306-314.

[12] G. Ma, H.C. Allen, DPPC Langmuir monolayer at the air-water interface: probing the tail and head groups by vibrational sum frequency generation spectroscopy, Langmuir 22 (2006) 5341-5349.

[13] M.K. Pawelec, T.R. Sosnowski, Langmuir-Wilhelmy balance studies of DPPC and CTAB films, in: K.A. Wilk (Ed.), Surfactants and Dispersed Systems in Theory and Practice, Vol. Oficyna Wyd. Polit. Wroc., Wroclaw, 2003, pp. 267-270.

[14] M.K. Pawelec, T.R. Sosnowski, Relaxation phenomena at the air-water interface with surfactants, Int. J. Appl. Mech. Eng. 8 (2003) 295-300.

[15] O. Albrecht, H. Gruler, E. Sackmann, Pressure-composition phase diagrams of cholesterol/lecithin, cholesterol/phosphatidic acid, and lecithin/phosphatidic acid mixed monolayers: a Langmuir film balance study, J. Colloid Interf. Sci. 79 (1981) 319-338.

[16] G. Kretzschmar, J. Li, R. Miller, H. Motschmann, H. Möhwald, Characterisation of phospholipid layers at liquid interfaces. 3. Relaxation of spreading phospholipid monolayers under harmonic area changes, Colloids Surf. A 114 (1996) 277-285.

[17] H. Yun, Y.-W. Choi, N.J. Kim, D. Sohn, Physicochemical properties of phosphatidylcholine (PC) monolayers with different alkyl chains, at the air/water interface, Bull. Kor. Chem. Soc. 24 (2003) 377-383.

[18] J.B. Li, J. Krägel, A.V. Makievski, V.B. Fainermann, R. Miller, H. Möhwald, A study of mixed phospholipid/ß-casein monolayers at the water/air surface, Colloids Surf. A 142 (1998) 355-360.
[19] J. Krägel, G. Kretzschmar, J.B. Li, G. Loglio, R. Miller, H. Möhwald, Surface rheology of monolayers, Thin Solid Films 284/285 (1996) 361-364.

[20] J. Krägel, J.B. Li, R. Miller, M. Bree, G. Kretzschmar, H. Möhwald, Surface viscoelasticity of phospholipid monolayers at the air/water interface, Colloid Polym. Sci. 274 (1996) 1183-1187.

[21] J. Li, R. Miller, H. Möhwald, Phospholipid monolayers and their dynamic interfacial behaviour studied by axisymmetric drop shape analysis, Thin Solid Films 284/285 (1996) 357-360.

[22] J. Li, R. Miller, D. Vollhardt, H. Möhwald, Spreading concentration effect on the morphology of phospholipid monolayers, Thin Solid Films 327-329 (1998) 84-86.

[23] J. Li, R. Miller, R. Wüstneck, H. Möhwald, A.W. Neumann, Use of pendent drop technique as a film balance at liquid/liquid interfaces, Colloids Surf. A 96 (1995) 295-299.

[24] H. Tournois, P. Gieles, R. Demel, J. de Gier, B. de Kruijff, Interfacial properties of gramicidin and gramicidin-lipid mixtures measured with static and dynamic monolayer techniques, Biophys. J. 55 (1989) 557-569.

[25] P. Dynarowicz-Łatka, K. Hąc-Wydro, Interactions between phosphatidylcholines and cholesterol in monolayers at the air/water interface, Colloids Surf. B 37 (2004) 21-25.

[26] J.M. Smaby, M.M. Momsen, H.L. Brockman, R.E. Brown, Phosphatidylcholine acyl unsaturation modulates the decrease in interfacial elasticity induced by cholesterol, Biophys. J. 73 (1997) 1492-1505.

[27] B.L. Stottrup, S.L. Keller, Phase behavior of lipid monolayers containing DPPC and cholesterol analogs, Biophys. J. 90 (2006) 3176-3183.

[28] B.L. Stottrup, D.S. Stevens, S.L. Keller, Miscibility of ternary mixtures of phospholipids and cholesterol in monolayers, and application to bilayer systems, Biophys. J. 88 (2005) 269-276.

[29] R. Miller, N. Wüstneck, J. Krägel, G. Kretzschmar, Dilational and shear rheology of adsorption layers at liquid interfaces, Colloids Surf. A 111 (1996) 75-118.

[30] J. Li, R. Miller, H. Möhwald, Characterisation of phospholipid layers at liquid interfaces. 2. Comparison of isotherms of insoluble and soluble films of phospholipids at different fluid/water interfaces, Colloids Surf. A 114 (1996) 123-130.

[31] G. Loglio, P. Pandolfini, R. Miller, A.V. Makievski, R. Ravera, M. Ferrari, L. Liggieri, Drop and bubble shape analysis as a tool for dilational rheological studies of interfacial layers, in: D. Möbius, R. Miller (Eds.), Novel Methods to Study Interfacial Layers, in Studies in Interface Science, vol. 11, Elsevier, Amsterdam, 2001, pp. 439-485.

[32] P. Saulnier, F. Boury, A. Malzert, B. Heurtault, T. Ivanova, A. Cagna, I. Panaiotov, J. Proust, Rheological model for the study of dilational properties of monolayers. Comportment of dipalmitoylphosphatidylcholine (DPPC) at the dichloromethane (DCM)/water interface under ramp type or sinusoidal perturbations, Langmuir 17 (2001) 8104-8111.

[33] G. Leneweit, M. Vrânceanu, K. Winkler, H. Nirschl, Surface rheology and phase transitions of phospholipid/cholesterol mixtures, Biophys. J., submitted for publication.

[34] G. Loglio, U. Tesei, R. Cini, Spectral data of surface viscoelastic modulus acquired via digital Fourier transformation, J. Colloid Interf. Sci. 71 (1979) 316-320.

[35] G. Loglio, P. Pandolfini, R. Miller, A.V. Makievski, J. Krägel, F. Ravera, B.A. Noskov, Perturbation-response relationship in liquid interfacial systems: non-linearity assessment by frequency-domain analysis, Colloids Surf. A 261 (2005) 57-63.

[36] G. Loglio, P. Pandolfini, R. Miller, A. Makievski, J. Krägel, F. Ravera, Oscillation of interfacial properties in liquid systems: assessment of harmonic distortion, Phys. Chem. Chem. Phys. 6 (2004) 1375-1379.

[37] http://www.vds.it/martin/eng/dati.htm.

[38] G. Loglio, P. Pandolfini, A.V. Makievski, R. Miller, Calibration parameters of the pendant drop tensiometer: assessment of accuracy, J. Colloid Interf. Sci. 265 (2003) 161-165.

[39] R.P. Woodward, Dynamic Surface Tension and Dilational Stress Measurements Using the Drop Shape Method, http://www.firsttenangstroms.com/.

[40] S. Decesari, M.C. Facchini, M. Mircea, F. Cavalli, S. Fuzzi, Solubility properties of surfactants in atmospheric aerosol and cloud/fog water samples, J. Geophys. Res. 108 (2003) 4685-4693. 
[41] I.N. Bronstein, K.A. Semendjajew, Taschenbuch der Mathematik, Verlag Nauka Moskau, Moskau, 1991.

[42] L. Caseli, D.C. Masui, R.P.M. Furriel, F.A. Leone, M.E.D. Zaniquelli, Adsorption kinetics and dilatational rheological studies for the soluble and anchored forms of alkaline phosphatase at the air/water interface, J. Braz. Chem. Soc. 16 (2005) 969-977.

[43] L. Lis, M. McAlister, N. Fuller, R. Rand, V. Parsegian, Measurement of the lateral compressibility of several phospholipid bilayers, Biophys. J. 37 (1982) 667-672.

[44] T.M. Okonogi, H.M. McConnell, Contrast inversion in the epifluorescence of cholesterol-phospholipid monolayers, Biophys. J. 86 (2004) 880-890.

[45] K. Nag, K.M.W. Keough, Epifluorescence microscopic studies of monolayers containing mixtures of dioleoyl- and dipalmitoylphosphatidylcholines, Biophys. J. 65 (1993) 1019-1026.

[46] L.A.D. Worthman, K. Nag, P.J. Davis, K.M.W. Keough, Cholesterol in condensed and fluid phosphatidylcholine monolayers studied by epifluorescence microscopy, Biophys. J. 72 (1997) 2569-2580.
[47] H. Möhwald, Phospholipid monolayers, in: R. Lipowski, E. Sackmann (Eds.), Structure and Dynamics of Membranes. From Cells to Vesicles, vol. 1A, Elsevier, Amsterdam, 1995, pp. 161-211.

[48] O. Albrecht, Polymorhismus in reinen und gemischten lipidmonoschichten, Thesis, University of Ulm, 1979.

[49] C. Yuan, L.J. Johnston, Phase evolution in cholesterol/DPPC monolayers: atomic force microscopy and near field scanning optical microscopy studies, J. Microsc. 205 (2002) 136-146.

[50] R. Wüstneck, P. Enders, T. Ebisch, R. Miller, Axialsymmetric stress relaxation and surface dilation rheology of docosanic acid monolayers spread at the interface of pendant drops in the short time region, Thin Solid Films 298 (1997) 39-46.

[51] P. Joos, M.v. Uffelen, G. Serrin, Surface relaxation in spread insoluble monolayers of cholesterol and dipalmitoyl lecithin, J. Colloid Interf. Sci. 152 (1992) 521-533.

[52] C.A. Helm, P. Tippmann-Krayer, H. Möhwald, J. Als-Nielsen, K. Kjaer, Phases of phosphatidyl ethanolamine monolayers studied by synchrotron X-ray scattering, Biophys. J. 60 (1991) 1457-1476. 